\title{
Dis-moi quel oral, et je te dirai quelle école...
}

Introduction

Introduction. As your oral practices, so your school

Introducción. Dime qué oral, y te diré qué escuela...

Daniel Coste et Roger-François Gauthier

\section{OpenEdition \\ Journals}

Édition électronique

URL : https://journals.openedition.org/ries/10360

DOI : $10.4000 /$ ries. 10360

ISSN : 2261-4265

\section{Éditeur}

France Education international

Édition imprimée

Date de publication : 1 avril 2021

Pagination : $57-68$

ISBN : 978-2-85420-629-6

ISSN : $1254-4590$

Référence électronique

Daniel Coste et Roger-François Gauthier, « Dis-moi quel oral, et je te dirai quelle école... », Revue internationale d'éducation de Sèvres [En ligne], 86 | avril 2021, mis en ligne le 01 avril 2022, consulté le 25 mars 2023. URL : http://journals.openedition.org/ries/10360 ; DOl : https://doi.org/10.4000/ries. 10360 


\title{
l'oral dans l'éducation
}

\section{Introduction}

\section{Dis-moi quel oral, et je te dirai quelle école...}

\author{
Daniel Coste $\mathrm{e}^{*}$ \\ École normale supérieure de Lyon \\ Roger-François Gauthier* \\ Inspecteur général honoraire
}

Le manque d'oral est considérable à tous les âges, dans toutes les strates de la société, à tout niveau de responsabilité.

Il crée bien des angoisses et des souffrances inutiles, gèle les talents sur place et bloque des évolutions professionnelles. Il bride l'expression citoyenne et de façon d'autant plus dommageable que les plus vulnérables en sont les premières victimes.

Cyril Delhay (2019)

Consacrer un dossier de la Revue internationale d'éducation à l'oral implique de reconnaître aussi bien l'intérêt que la spécificité de ce sujet: s'il est relativement stimulant d'interroger les systèmes d'éducation d'une dizaine de pays, de quatre continents choisis pour la diversité qu'ils laissaient attendre, à partir d'un questionnement sur l'oral, il faut avoir en tête que ce que l'on appelle oral, d'un pays à l'autre, d'une culture scolaire à une autre est si différent que la comparaison, à laquelle conduit nécessairement cette revue, doit être en ce cas particulièrement prudente.

La première remarque est qu'aucun des pays évoqués ici ${ }^{1}$ ne décrit la situation de l'oral en éducation comme un objet stabilisé: la prise en compte de l'oral à l'école "bouge ", partout, et même dans les cas où l'intérêt pour l'oral est actuellement moins développé, l'idée est qu'existe un mouvement irrépressible et d'origines diverses. On peut s'en étonner, tant dans certains pays, comme la France, l'oral - et plus encore, au sein de l'oral, la compétence à « écouter » - apparaît un peu comme le parent pauvre des efforts d'une école qui privilégie classiquement l'écrit. S’il ne saurait être question de dresser ici un palmarès de l'« oratie ${ }^{2}$ » entre les pays observés, la lecture de ce dossier permet de prendre conscience de la complexité de la question et de la richesse de travaux conduits ici ou là.

\footnotetext{
* Daniel Coste et Roger-François Gauthier tiennent à remercier Patrick Rayou pour le concours qu'il a apporté à la conception et à l'élaboration de ce dossier.

1. Angleterre, Argentine, Côte d'Ivoire, France, Inde, Italie, Japon, Suisse, Tunisie.

2. Voir plus loin la définition proposée par les auteurs (NdIR).
} 


\section{UN NUANCIER CONTRASTÉ PAR L'HISTOIRE DES RELATIONS ENTRE L'ORAL ET L'ÉCOLE}

Les rapports sont spécifiques et complexes dans toutes les traditions scolaires et nationales entre l'oral et l'école. L'article de G. N. Devy, traitant de la longue prééminence culturelle de l'oral en Inde, mais aussi l'article de Michel Tozzi pour la France, se référant à l'apologie socratique de l'oral, nous rappellent que l'enseignement fut partout d'abord oral, en référence à de grandes œuvres orales, et avec des activités orales, qu'il se soit agi selon les cas du «par cœur » dans ses excès ou de la réflexion philosophique.

Toutefois, les systèmes scolaires qui se sont inscrits dans le contexte du développement de l'imprimerie comme des États-nations ont consacré le règne de l'écrit et la minoration de l'oral comme objet d'enseignement: l'article indien montre cette "sortie de l'oral ", celui de Mokhtar Sahnoun, à propos du contexte tunisien, relève bien l'état actuel de cette minoration, quand l'article italien, dû à Miriam Voghera, rappelle à quel point la langue italienne fut longtemps une réalité seulement écrite. L'article français souligne que jusqu'à une date récente, l'oral à l'examen du baccalauréat se voyait attribuer la qualification de « de rattrapage »!

Puis, évolution commune à tous les pays abordés, bien sûr selon des calendriers et des modalités diverses, on a assisté à une prise de conscience de cette minoration de l'oral dans la formation des enfants et on a souhaité y remédier: la Suisse, dont la situation est ici décrite par quatre auteurs (Roxane Gagnon, Stefan Hauser, Sonia Guillemin et Rosalie Bourdages) aussi bien pour la partie alémanique que pour la Romandie, mais plus encore le Japon, analysé dans l'histoire longue par Nozomi Takahashi, nous montrent ici la profondeur de cette reconquête, et jusqu'à un certain point l'Italie, même si Miriam Voghera nous rappelle l'écart entre le curriculum théorique et la réalité. La France elle-même a fait preuve de façon un peu ponctuelle d'innovation en créant, à partir de 2021, une épreuve importante dite « grand oral » à l'examen du baccalauréat.

C'est ainsi que l'oral est devenu un objet de plein droit pour la réflexion en éducation. Les remarquables notes de synthèse ou bilans établis par Elisabeth Nonnon $(1999,2016)$ pour ce qui est du domaine francophone, ou encore par Marie Gaussel dans un dossier de veille de l'IFE (2017) en témoignent à l'évidence.

Cette " ouverture à l'oral " correspond pourtant à des contextes politiques très divers. Le fait qu'en Angleterre, une commission parlementaire se penche sur la question en 2020-2021, sous l'intitulé significatif Speak for Change, est à cet égard particulièrement notable. Ailleurs, beaucoup dépend de quelle instance décide de quoi à quelle échelle de responsabilité. Dans le cas de l'Argentine, analysé ici par Constanza Padilla, c'est l'extrême fragmentation du système éducatif qui est soulignée, reflet de l'organisation fédérale de l'État. Et la situation de l'Inde multilingue et de ses 29 États est tout aussi révélatrice.

Pour cette dernière comme pour le Japon, l'histoire au long cours est convoquée par les auteurs pour marquer la transformation des rapports entre oral et écrit dans la durée. Mais là où l'Inde se caractérise par une évolution que scande 
l'extension progressive de la reconnaissance officielle de langues écrites, dépossédant en partie l'oral de sa fonction de transmission, c'est l'unification lente du japonais comme langue nationale, dans la relation entre mode d'écrire et mode de dire, qui caractérise la situation. Les héritages historiques, au sens large, ont aussi une incidence forte sur la situation actuelle de l'oral en Tunisie ou en Côte d'Ivoire, pays analysé ici par Bénédicte Techti.

Le contexte de cette évolution générale, même si elle correspond à des niveaux de prise en compte très variables, est celui des évolutions technologiques, qui donnent à l'oral des dimensions nouvelles, mais aussi celui des mouvements de population, des dimensions transculturelles d'une certaine mondialisation, qui modifient, pour la plupart des systèmes éducatifs, les questions que leur pose l'oral et le traitement qu'ils lui réservent.

Parmi les facteurs qui pèsent favorablement sur le développement de la prise en compte de l'oral à l'école, figure la prise de conscience par la plupart des sociétés de son importance en dehors même de l'école. Une demande sociale vient d'abord du monde du travail, en raison notamment de l'extension du secteur des services et de la tertiarisation de nombreuses fonctions, où l'expression orale du collaborateur, dès l'embauche, est importante.

Diverses préoccupations de bien des sociétés, en direction par exemple de la recherche du développement personnel, ou de ce que l'article japonais appelle la " capacité à vivre ", jouent un rôle aussi pour la revalorisation de l'oral et la demande adressée en ce sens à l'école. L'oral fait désormais partie de ces "compétences du $\mathrm{XXI}^{\mathrm{e}}$ siècle » ou "life skills», qui sont au programme des réformes curriculaires de nombreux pays.

Dans le monde civil et politique, de la même façon, la valorisation sincère ou feinte de la démocratie a pour conséquence que de nombreuses collectivités demandent à l'école de contribuer au fonctionnement harmonieux des sociétés et au développement de cette démocratie: ce fut le cas au Japon au $\mathrm{xx}^{\mathrm{e}}$ siècle, c'est le cas en Tunisie depuis la « révolution ». C'est aussi ce que l'on attend en France de la discussion à visée philosophique ou de la pratique du débat, et c'est aussi en Côte d'Ivoire que l'institution scolaire aimerait, en développant l'oral, agir contre les traditions du silence imposé aux enfants. La question ici est de savoir à la fois quelle mesure prennent les différents pays de cette évolution en direction d'une plus grande ouverture de l'école à l'oral, et comment ils tentent de répondre aux défis que présente l'oral.

Avant de rechercher quelles formes scolaires peut prendre l'oral tel qu'il se développe, il y a lieu de rappeler son caractère paradoxal, car c'est sur fond de ces caractéristiques que les mouvements en cours s'inscrivent.

\section{L'ORAL, UN OBJET PARADOXAL}

\section{Un oral omniprésent, inaperçu et ancillaire}

Dans la mesure où l'école est, par excellence, le lieu marqué par l'apprentissage de l'écrire et du lire, par l'accès écrit aux connaissances et aux ouvres, le 
recours qu'elle fait au parler et à l'écouter apparaît souvent comme simplement instrumental, indispensable certes, mais ancillaire. L'oral va de soi et « va sans dire ». Il n'est pas perçu comme un savoir à maîtriser pour lui-même mais comme « un préalable au service de la culture scolaire » (Langlois, 2012).

\section{L'oral, ça s'attrape ou ça s'apprend?}

Pour nombre des enseignants anglais répondant à une enquête de 2016, dont rend compte Will Millard, "l'oral, ça s'attrape » autant ou plus que "ça s'apprend ». Nul besoin, dès lors, d'en faire un objet d'apprentissage en soi. "Écouter/ parler» ne fait pas partie des trois compétences de base: lire, écrire, compter. On réserve l'attention pour l'oral à l'apprentissage des langues étrangères.

\section{L’oral, ça se contrôle}

Avec les meilleures intentions du monde et dans l'intérêt même des élèves, le rôle de l'école reste souvent pensé comme de brider l'oral et de le rectifier. Peu d'articles abordent ce point, hormis celui que Mokhtar Sahnoun consacre à la situation tunisienne ou celui de Bénédicte Techti sur la Côte d'Ivoire. Sujet devenu tabou et politiquement incorrect ailleurs?

\section{L'oral, ça fait peur}

Le contrôle de la parole des élèves n'est pas seulement affaire de correction de la langue, mais aussi de respect de l'ordre et de la discipline. Comme le déclarent certains des enseignants dans l'enquête anglaise, à trop laisser les élèves parler entre eux, il y a risque d'encourager au relâchement. Les témoignages ne manquent pas d'enseignants qui, dans des classes "difficiles", ont effectivement peur de perdre toute autorité et de faillir à leur devoir s'ils laissent par trop les élèves s'emparer de la parole.

Mais, ailleurs, c'est aux élèves que l'oral fait peur. Répondre à une question de l'enseignant, passer au tableau, réciter un poème devant les autres, se résume à autant d'épreuves qui peuvent être source de malaise.

\section{L'oral, ça engage le corps et on peut y perdre la face}

Dans les textes suisse et anglais, cette incarnation de l'oral est mise en avant dans sa matérialité audible et visible. L'oral, c'est une voix, un timbre, une intonation et un rythme, mais aussi des gestes, des mimiques, une certaine tenue de l'ensemble du corps, une distance ou une proximité physique, le tout s'inscrivant à la fois dans un jeu de codes sociaux et culturels variables suivant les contextes et dans une interprétation personnelle - consciente ou non - de ces codes.

La dimension identitaire de l'oral peut aussi être affirmée, revendiquée (ou à l'inverse stigmatisée, discriminée) au niveau non pas d'un individu mais d'une communauté. Et cette valorisation ou cette dévalorisation (d'une langue régionale ou minoritaire, d'un « accent » particulier, d'une forme de " parler jeune ») ont avant 
tout l'oral pour objet (Blanchet 2016, 2019; Blanchet \& Clerc Conan, 2018). Cela concerne aussi l'école quand elle entend contribuer à un travail de standardisation, normalisation, unification nationale.

Certains, les mieux avertis et les mieux armés, recherchent les formations, le coaching, l'exercice guidé de leurs compétences et prestations orales ou encore, comme le souligne la contribution anglaise, s'assurent que leurs enfants fréquentent des établissements où existent des occasions de développer ce type de maîtrise de l'oral. Serait-ce que l'oral, décidément, s'apprend et se travaille?

\section{L'oral qui s'apprend: vers la définition d'une « oratie »?}

Trois autres points sont à établir, si l'on veut s'avancer vers la définition d'un enseignement de l'oral.

1) L'oral qui s'apprend n'est pas seulement celui prêt à dire, du last draft et du copy ready, évoqués dans la contribution anglaise. C'est aussi celui qui cherche et se cherche, qui explore (exploratory talk) et peine à le faire: l'oral des reprises et des impasses, des reformulations et des hésitations, celui qui a besoin de temps pour exprimer ce qu'il veut dire, le brouillon d'oral qui, à l'antenne, impatiente les grands médias de communication et que le temps de la classe ne tolère guère plus.

2) Le brouillage des frontières entre oral et écrit: le temps n'est plus où on pouvait latiniser à peu de frais avec l'adage verba volant scripta manent. L'oral s'inscrit dans les échanges que permettent les écrits des réseaux sociaux, les chats, il s'y insinue et y importe et fait évoluer ses propres modes de transcription, il s'ouvre à des emprunts à d'autres langues et les mêle à loisir. La liste est longue de ces proliférations et de ces brouillages, auxquels les élèves, même très jeunes, sont exposés et peuvent euxmêmes d'adonner.

3) La pluralité des répertoires langagiers dans les classes vient compléter et compliquer cet ensemble de pratiques multimodales des jeunes. Dans des contextes aussi différents que ceux de l'Italie, de la Tunisie, de l'Inde, de la Suisse, de la Côte d'Ivoire, la présence de langues régionales, de dialectes, de variétés endogènes de la langue majeure de scolarisation pose des questions de norme, de contact, d'alternance, de domination, de décalage entre l'officiellement prescrit et ce qui est pratiqué dans les faits.

Au bout du compte, ce n'est pas un hasard si, dans les articles comme dans cette présentation générale, on relève des variations de désignation entre " oral », " oralité », " pratiques orales ", " écouter et parler », " compréhension orale et expression orale »... Variations et parfois hésitations renforcées quand il s'est agi de traduire pour ce dossier des textes d'abord écrits en espagnol, en italien ou en anglais. On a même pu s'interroger sur l'introduction d'un néologisme tel que " oratie » qui apparaît dans le texte anglais à propos du rapport Oracy: The State of Speaking in our Schools. De fait, il semble pertinent de proposer « oratie » en français 
car, s'ajoutant bien sûr à « littératie » et à " numératie », ce mot place l'oral à hauteur de l'écrit et de la capacité mathématique dans l'équipement de base que l'école doit garantir.

\section{UNE ORIENTATION PRAGMATIQUE DES CURRICULUMS}

\section{Une véritable programmation de l'oral}

Là où un changement apparaît, dans certains pays, c'est d'abord dans le fait que l'oral donne lieu à programmation dans les instructions ou recommandations institutionnelles. La Suisse en fournit un exemple très clair avec le développé des contenus et des attendus en matière d'apprentissage de l'oral, tant pour le Lehrplan de Suisse alémanique que pour le Plan d'études romand (PER). L'oral y est appréhendé en termes de compétences visées, elles-mêmes illustrées par des descripteurs de savoir-faire. On retrouve là des approches de type pragmatique qui ont été retenues et illustrées, tout particulièrement pour l'apprentissage des langues étrangères, par les échelles de niveaux du Cadre européen commun de référence pour les langues (CECRL), publié en 2001.

La caractérisation des attendus aux différents cycles de la scolarisation apparaît aussi dans les articles consacrés à l'Italie ou à l'Argentine. Des descripteurs de cet ordre sont par ailleurs mentionnés dans la contribution portant sur la Côte d'Ivoire, à propos du Socle commun de référence ivoirien (SCRI). Dans ce dernier cas, ils se trouvent pour le moins en décalage sensible avec le quotidien des échanges dans les classes. Transfert indu et abusif de technologie éducative ou orientation réaliste et porteuse d'avenir dans des contextes aussi complexes?

\section{La montée de l' " écouter »}

La reconnaissance du parler s'est souvent faite au prix d'un oubli de l'écouter. Ce n'est plus le cas dans les indications curriculaires que comportent les articles des pays qui en proposent. L'écoute y figure de manière développée et dans l'ensemble de ses dimensions. Pour n'en prendre qu'un court exemple, cet extrait de l'article italien de Miriam Voghera, à propos de ce qui est prévu pour le premier cycle du secondaire:

En ce qui concerne l'écoute, voici quelques-uns des objectifs principaux: a) écouter des textes produits par d'autres, y compris transmis par les médias, en en reconnaissant la source et en identifiant le but, le sujet, les informations principales et le point de vue de l'émetteur; b) écouter des textes en appliquant des techniques qui étayent la compréhension: pendant l'écoute (prise de notes, mots-clés, courtes phrases de synthèse, signes conventionnels) et après l'écoute (retravailler les notes, expliciter les mots-clés, etc.).

De façon complémentaire, faciliter l'écoute, c'est aussi, pour les élèves (et pour les enseignants), travailler sa propre voix, contrôler son élocution, son souffle, ses gestes accompagnant la production de l'oral. 
Cette capacité d'écoute s'appuie sur l'écoute de dialogues ou d'interventions monologiques longues (Suisse alémanique), la variation selon les genres et les situations (Suisse romande), l'attention portée non seulement à ce qui est dit mais aux éléments paralinguistiques, voire aux émotions, aux visées non explicitées (Italie). Il s'agit bien de travailler sur la pluralité des oraux, avec des possibilités de retour réflexif sur les stratégies d'écoute qui ont été mobilisées (Suisse alémanique), mais aussi, notamment dans la formation universitaire, de donner à analyser des corpus de transcription d'échanges oraux (Tunisie).

\section{Traiter la question des (ir)régularités langagières à l'oral}

L'article sur l'université tunisienne est révélateur de tensions entre les pratiques de l'oral, les analyses qu'on peut en faire et, d'un autre côté les dimensions grammaticales, orthographiques et autres, descriptives ou normatives, en relation aux régularités des systèmes linguistiques. Il n'y a que peu de mentions de cette tension dans les contributions à ce dossier, mais ce n'est pas un hasard si elles figurent, sous une forme ou une autre, à propos des contextes où il $\mathrm{y}$ a, dans le système éducatif et dans les classes, contact de langues, de variétés linguistiques d'une même langue (Tunisie, Côte d'Ivoire), de dialecte et standard (Suisse alémanique, Italie).

Cet oral «débridé » n'entrant pas dans les normes habituelles de la description grammaticale ou de l'adéquation lexicale, s'il n'est pas frontalement abordé comme tel, est toutefois signalé en creux sous trois aspects. D’abord, comme juste noté, dans la mise en contraste, dans la formation de spécialistes de français, entre les cours portant sur le système linguistique de la langue et l'observation de transcriptions d'échanges ordinaires entre jeunes dans une variété locale (Tunisie). Ensuite, à travers l'importance accordée à l'apprentissage et à l'enrichissement du vocabulaire, l'oral spontané des élèves étant réputé pauvre quant au lexique (Suisse alémanique, Angleterre). Enfin, par une approche des régularités de l'oral au niveau des genres discursifs, grâce à des modèles d'analyse transversaux permettant de décrire, en utilisant les mêmes catégories, discours écrits et discours oraux (Suisse romande).

\section{Quel oral en classe?}

Dans l'article sur l'Angleterre, rappel est fait de la typologie de teaching talk proposée par Robin Alexander, qui distingue cinq manières, pour l'enseignant, de gérer dans la classe l'oral des élèves, depuis le fait de transmettre des connaissances en demandant aux élèves une restitution "par cœur » d'éléments clés, jusqu'à une conduite dialogique recourant à une forme de discussion nourrie par des questionnements structurés, afin d'aider les élèves à approfondir leur compréhension des connaissances, des principes et des processus fondamentaux. Ce dernier type est distingué de la simple discussion qui voit l'enseignant encourager les élèves à échanger des idées et des informations. 
Dans cette liste, Alexander ne mentionne pas comme tel le débat, mais ce genre particulier est cité à plusieurs reprises dans le reste de l'article, de même que dans l'article consacré à la Suisse et, plus brièvement, dans certaines des autres contributions (Italie, voire Japon).

La contribution japonaise montre, de façon différente, comment, dans une certaine durée historique, mais avec une accélération récente, les désignations - disons occidentales - des activités orales (exposé, discussion, etc.) ne sauraient convenir à caractériser les fonctions et les représentations attachées aux usages de l'oral quand la langue ne devient que peu à peu matière scolaire et quand son apprentissage relève plus d'une morale puis d'une forme d'affirmation nationale que d'une visée pragmatique ou cognitive. Mais, au cours des dernières décennies, c'est à un mouvement accéléré de référence - sinon toujours de recours - à des caractérisations en quelque sorte importées qu'on a pu assister, y compris avec l'emprunt et la japonisation du terme « communication».

\section{L'oral pour apprendre}

Une forme de consensus se dégage de l'ensemble des articles: l'oral s'apprend et doit s'enseigner. Ne pas le faire, c'est contribuer au maintien ou pire, au renforcement des inégalités entre les élèves. Autre point de large convergence, explicitée ou non: c'est aux enseignements et aux enseignants de langue de répondre au besoin de travailler l'oral. Cela relève prioritairement de leur responsabilité; responsabilité que les enseignants d'autres disciplines leur délèguent bien volontiers. Là encore, l'enquête de 2016 auprès d'enseignants anglais établit clairement ce constat.

À revenir toutefois sur les types d'activités en classe et leur mode de désignation, certaines contributions se caractérisent aussi par le rapport à établir entre l'oral et la construction de connaissances. Cette attention portée à un oral pour bien ou mieux apprendre se trouve surtout dans trois lieux et sous des intitulés pourtant bien distincts: le cinquième type de la classification d'Alexander, déjà mentionné; la «discussion à visée philosophique » que présente la contribution française; le «modèle dialogique d'enseignement » retenu pour certaines recherches en Argentine. Dans chacune de ces approches, inscrites dans des contextes, à des niveaux et pour des publics différents, l'oralité est pensée comme au service de l'élaboration et de l'appropriation "de connaissances, de principes et de processus » (Alexander, 2020).

C'est dans une perspective explicitement socioconstructiviste qu'on se situe alors, inspirée de Vygotski, et qui souligne la dimension épistémique de l'oralité, comme le montre l'article sur l'Argentine. Mais se marque aussi le rôle central de l'enseignant «dans un contexte qui favorise la construction partagée d'apprentissages, avec une médiation enseignante précise, systématique et graduelle».

Cet oral paradoxal dans l'éducation, omniprésent et inaperçu, c'est aussi celui qui non seulement renvoie à une oralité multiforme, d'une oratie au sein d'une littératie étendue, mais peut aussi jouer, dans cette configuration, un rôle moteur au service, non plus de l'écrit, mais de l'ensemble des apprentissages scolaires. 


\section{L'ORAL À CERTAINES CONDITIONS}

S’il semble souhaitable aux communautés éducatives de renforcer la place de l'oral dans l'éducation, un certain nombre de conditions sont souvent rappelées.

1) Une formation adaptée des enseignants est indispensable, et semble avoir été négligée par certains pays par ailleurs volontaires pour développer la place de l'oral, comme l'Italie: il s'agit non seulement de mieux former les enseignants à une fonction qui est essentiellement orale, mais aussi d'en faire de véritables pédagogues de l'oral dans ses trois dimensions pour l'élève: pour favoriser aussi bien son propre développement psychique, que son relationnel aux autres, ou le développement de ses connaissances dans tous les domaines grâce à l'oral.

2) Un regard attentif doit être porté sur le rapport entre enseignement de l'oral et l'équité, sujet controversé: les articles du dossier représentent autant l'idée que l'oral peut donner des chances à des élèves qui sont peu à l'aise avec l'écrit, que celle selon laquelle l'oral renforce les inégalités, notamment sociales, motif pour lequel les Anglais ont retiré l'oral des épreuves du GCSE${ }^{3}$.

3) Il est important de ne pas renoncer à évaluer l'oral, sinon il restera proche du statut de parent pauvre, tout en évitant les dérives possibles de toute évaluation vers des aspects formels éloignés de la richesse de l'oral et en prenant garde au coût, en termes de temps, d'épreuves de production orales quand elles doivent être programmées de façon successive.

4) La meilleure prise en compte de l'oral passe aussi par un autre aménagement des espaces scolaires, une autre disposition des classes, avec des effectifs moindres et des équipements adéquats et disponibles, ainsi que des ressources, notamment en matériels enregistrés pour favoriser l'écoute diversifiée.

Bien sûr, on observe en effet des variations significatives des chances de mise en place d'un enseignement de qualité de l'oral selon les contextes. Peu favorables sont les cas où les modèles et les attendus des curriculums sont en quelque sorte importés comme relevant d'une technologie éducative dominante et circulante mais totalement « hors sol », au regard des réalités de terrain.

Plus favorables sont les cas, comme la Suisse romande le montre, où il y a conjonction apparente entre différentes dimensions d'une évolution des pratiques touchant à l'enseignement-apprentissage de l'oral (non isolément) et, de fait, mise en œuvre d'un processus curriculaire au plein sens de la notion de curriculum, non réduit à un programme institutionnel. Existe notamment une coopération entre enseignants-chercheurs-formateurs d'une Haute École pédagogique (institut de recherche et de formation des enseignants) et des équipes universitaires de chercheurs en sciences de l'éducation et du langage. Ces derniers sont eux-mêmes impliqués, d'une part, dans la réflexion sur la didactique des discours et, d'autre part, avec leurs collègues de la HEP, dans le suivi de démarches d'enseignement

3. General Certificate of Secondary Education, examen intervenant à la fin du secondaire inférieur. 
telles que celles des « séquences didactiques » dont l'article fait état et qui ont donné lieu à la mise au point d'outils pédagogiques diffusables. Il ne s'agit pas d'ériger en modèle ce dispositif mais de souligner que l'apprentissage de l'oral, dans une perspective de rénovation des cursus d'études, suppose l'intervention, au contact des lieux d'enseignement et des lieux de formation, de membres complémentaires d'équipes partenaires, assurant, à titre d'expérience limitée dans un premier temps, les différentes phases d'une innovation curriculaire. Le cas de l'Argentine est aussi très significatif à cet égard, mais en reste plus à des expériences ponctuelles dans le cadre de projets de recherche.

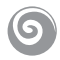

De ce parcours dans quelques contextes nationaux et dans diverses formes de rapport à l'oral, oralité ou oratie, que conclure? Avant tout, qu'il n'a pu porter qu'un éclairage très partiel sur un objet qui, de la répétition psalmodiée à l'improvisation chahutée, de la discussion orientée à l'allocution théâtralisée, donne lieu à déclinaisons multiples entre lesquelles, au-delà de l'évidence du rapport entre une parole et une écoute, toute généralisation s'avère délicate. Si toutefois on s'y risque, quelques constats et perspectives se dégagent de cette mise en regard internationale.

L'oral n'est plus passé sous silence. Il se fait entendre. On en parle et il est reconnu comme un enjeu éducatif majeur. On s'inquiète de savoir comment parler, comment écouter. Si on reprend une définition que, dans une société de l'information, l'OCDE donne de la littératie: «l'aptitude à comprendre et à utiliser l'information écrite dans la vie courante, à la maison, au travail et dans la collectivité en vue d'atteindre des buts personnels et d'étendre ses connaissances et ses capacités ", on voit bien que l'oral coche toutes les cases et vaut qu'on risque le terme d'oratie et qu'on en étende la portée au-delà du traitement et de l'usage de l'information.

L'oral, ça s'apprend et ça mérite un enseignement. Et donc, où qu'on soit et avec une bonne part de variation selon les contextes, il y faut des contenus, des supports, des méthodes, des normes, des évaluations. Si l'école ne s'en charge pas, cela se passe ailleurs, mais pas pour tous. Les inégalités demeurent ou se creusent, les discriminations se renforcent. Enseigner la parole, c'est aussi donner les moyens de la prendre dans l'espace public et la vie sociale.

En filigrane encore et demandant affirmation plus nette: l'oral, c'est l'affaire de tous et de toutes les matières. Car, dans le processus éducatif comme dans des sociétés plurielles et technologisées, l'oral se démultiplie en genres et en pratiques diversifiés. Dès lors que l'enjeu n'est pas seulement d'apprendre à se servir de la parole mais aussi de se servir de la parole pour apprendre, chaque discipline y trouve son intérêt et a à y prendre ses responsabilités. C'est à cette condition aussi que l'oral, longtemps seul vecteur majeur de transmission des connaissances et des valeurs, peut retrouver sa pleine place dans leur construction participative, en interjeu avec l'écrit et d'autres modes de communication.

$\mathrm{Au}$ bout du compte, en revenir au titre de cette présentation: "Dis-moi quel oral, et je te dirai quelle école... » 


\section{BibLIOgRAPHIE}

ALEXANDER R. (2020). A Dialogic Teaching Companion. London: Routledge.

BLANCHET P. (2 éd.: 2019). Discriminations: combattre la glottophobie. Limoges:

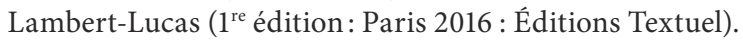

BLANCHET P., CLERC CONAN S. (2018). Je n’ai plus osé ouvrir la bouche... Témoignages de glottophobie vécue et moyens de se défendre. Limoges: Lambert-Lucas.

DELHAY C. (2019). Baccalauréat 2021. Faire du grand oral un levier d'égalité des chances: recommandations pour le grand oral du baccalauréat et l'enseignement de l'oral, de l'école maternelle au lycée. Rapport remis à Jean-Michel Blanquer, ministre de l'éducation nationale et de la jeunesse, le 19 juin 2019. Paris: Ministère de l'éducation nationale et de la jeunesse, juin 2019. En ligne: https://bit.ly/314PHkd/

GAUSSEL M. (2017). Je parle, tu dis, nous écoutons: apprendre avec l'oral. Dossier de veille de l'IFE, $n^{\circ} 117$, avril. Lyon: ENS de Lyon. En ligne: https://bit.ly/3tFYeX2

LANGLOIS R. (2012). Les précurseurs de l'oralité scolaire en Europe: de l'oral à la parole vivante. Mont-Saint-Aignan: Publications des universités de Rouen et du Havre.

NONNON É. (1999). «L'enseignement de l'oral et les interactions verbales en classe: champs de référence et problématiques ». Revue française de pédagogie, nº 129, p. 87-131. En ligne: https://bit.ly/3tJAMZ6/

NONNON É. (2016). « 40 ans de discours sur l'enseignement de l'oral: la didactique face à ses questions ». Pratiques, n 169-170, n.p. En ligne: https://journals.openedition.org/ pratiques/3115/

WEBER C. (2013). Pour une didactique de l'oralité. Enseigner le français tel qu'il est parlé. Paris: Didier. 
